\title{
La géotraçabilité dans le secteur vitivinicole : construction institutionnelle du marché
}

Anne Isla

\section{CpenEdition}

1 Journals

Édition électronique

URL : https://journals.openedition.org/rei/6079

DOI : 10.4000/rei.6079

ISSN : 1773-0198

Éditeur

De Boeck Supérieur

\section{Édition imprimée}

Date de publication : 30 mars 2015

Pagination : 73-102

ISBN : 9782804193607

ISSN : 0154-3229

\section{Référence électronique}

Anne Isla, "La géotraçabilité dans le secteur vitivinicole : construction institutionnelle du marché », Revue d'économie industrielle [En ligne], 149 | 1er trimestre 2015, mis en ligne le 30 mars 2017, consulté le 02 juin 2022. URL : http://journals.openedition.org/rei/6079 ; DOI : https://doi.org/10.4000/rei.6079 


\title{
LA GÉOTRAÇABILITÉ DANS LE SECTEUR VITIVINICOLE : CONSTRUCTION INSTITUTIONNELLE DU MARCHÉ
}

\author{
Anne Isla, LEREPS, Université de Toulouse
}

Mots clés : Convention de qualité, institutions, régulation, encastrement social, secteur du vin, traçabilité, logiciel d'analyse textuelle.

\section{Keywords: Quality Convention, Institutions, Regulation, Social Embededdness, Wine Sector, Traceability, Textual Analysis Software.}

La volonté de tracer le vin s'inscrit au croisement de plusieurs mutations qui marquent l'évolution du marché aujourd'hui : i) un mouvement actuel de développement de la traçabilité des produits, que ceux-ci soient alimentaires ou non ; ii) une généralisation depuis quelques années des $A O C^{I}$ qui fait perdre de leur crédibilité à cette marque collective (Smith et al., 2007) ou à ce bien de club (Torre, 2002) et qui peut conduire les producteurs à rechercher des solutions pour se démarquer en fournissant une information supplémentaire au consommateur ; iii) une évolution dans ce que Barrère (2007) appelle le paradigme du goût - le paradigme du marché de masse du goût remplaçant le paradigme aristocratique ou, dit autrement, le goût devenant affaire de tous entraîne une mutation fondamentale dans la segmentation du marché vitivinicole et une exigence pour les producteurs d'« éduquer » le consommateur ; iv) une inquiétude face à

I AOC, Appellation d'Origine Contrôlée en France, devenue depuis 2008 Appellation d'Origine Protégée, AOP, en Europe. Pour cette raison, nous parlerons d'AOP et non d'AOC dans la suite du texte. 
l'évolution de la réglementation, la fusion, en 2009, des 2I $\mathrm{OCM}^{2}$ agricoles européennes dans une OCM unique entraînant diverses mutations, aussi bien dans la segmentation du marché vitivinicole que dans l'étiquetage ; v) un secteur dont certains segments sont en crise depuis la fin des années I990 avec une globalisation du marché et une concurrence de plus en plus importante des vins du Nouveau Monde.

Dans ce contexte, tracer le vin est, pour le producteur, la volonté de créer du lien avec le consommateur, de donner du sens à l'AOP et de transformer le consommateur en connaisseur en lui donnant le sentiment qu'il entre dans le cercle des amateurs éclairés de vin. Sensibiliser le consommateur aux enjeux de la qualité (terroir, etc.) et au-delà de la protection de la nature et de l'éthique du travail devient ainsi un facteur clé de la durabilité du marché du vin. La géotraçabilité du vin répond à ces exigences, elle envisage d'ajouter aux informations sur la traçabilité des géo-indicateurs : localisation de la parcelle, nature du sol, données climatiques, exposition du vignoble, etc. Concrètement, le consommateur peut scanner à l'aide de son téléphone le datamatrix ${ }^{3}$ ou entrer le numéro inscrit sur la bouteille pour avoir accès à un portail internet spécifique et retrouver toutes les informations relatives au vin et à sa provenance.

Le producteur ouvre ainsi un espace d'interactions avec le consommateur, ce qui lui permet notamment de configurer lui-même l'information qui lui convient ${ }^{4}$. Il s'agit de modifier à son avantage l'organisation du marché. Comme le souligne Moati pour les marques de distributeurs, ce n'est rien moins qu'une remise en cause de l'architecture des marchés de masse. Ce n'est rien moins qu'une définition de la qualité du produit. L'enjeu est l'adaptation de l'offre aux attentes des consommateurs. La clientèle devient un patrimoine ${ }^{5}$ qu'il s'agit de protéger et de valoriser grâce à politiques de fidélisation qui cherchent à diversifier les relations entre l'offre et la demande (Moati, 20IO, p. I49). La géotraçabilité doit aussi intégrer les nouvelles exigences réglementaires en matière de traçabilité et d'étiquetage et

2 Organisation commune du marché.

3 Code-barres à deux dimensions.

4 Nous avons notamment étudié le projet Geowine de traçabilité du vin mis en place par des producteurs du Sud-Ouest français, cf. http://www.geowine.net/

5 Nous reviendrons plus loin sur cette conception qui rejoint le concept de " convention de qualité domestique ». 
être adaptable à celles qui pourraient émerger dans le futur. C'est ce cadre institutionnel réglementaire qui concentre la réflexion de notre article. "As sets of norms they not only place limits on industrial practices (e.g. environmental standards [...]) but also set boundaries on markets (e.g. the definition of what constitutes wine) "(Jullien et Smith, 20II, p. 37I). Nous considérons donc l'évolution de la réglementation comme encastrée dans les processus d'évolution sociale. Ce dont il est question, c'est de définir les conventions de qualité qui seront tenues comme légitimes, pendant une période, dans un espace géographique donné.

Parce que la convention définit, à un moment donné, les formes légitimes de production et d'échange (Salais et al., 1986), nous mobiliserons le cadre théorique conventionnaliste. Il y a entente sur la convention de qualité qui sera retenue comme légitime. Cette entente se concrétisera notamment dans le système juridique mis en œuvre. La définition théorique des conventions de qualité et leur instrumentalisation par le droit seront donc l'objet de notre première partie. La seconde partie illustrera l'évolution des conventions de qualité vitivinicole en Europe depuis les années I960 à travers l'évolution du cadre institutionnel. Cette analyse est outillée par un logiciel de traitement statistique de textes. Ce logiciel permettra de montrer (de façon automatique et objective) les saillances (Schelling, I960), les points forts du chemin d'évolution du cadre institutionnel.

\section{CONVENTION DE QUALITÉ, QUALITÉ DU VIN ET RĖGLE DE DROIT}

Lorsque le cadre institutionnel (the operationnal rules de Commons) est perçu comme changeant, les acteurs peuvent considérer qu'il s'agit d'une opportunité, et plutôt que de se rabattre sur l'habitude, ils vont répondre de façon créative à la situation telle qu'ils l'évaluent. L'enjeu est la construction de nouvelles pratiques qui pourraient être institutionnalisées dans l'évolution de la réglementation, donnant son dynamisme à la société capitaliste, son principe de changement sans fin (Commons, I934, p. I04). Les projets de géotraçabilité s'inscrivent dans cette perception d'un monde changeant. Ils combinent un projet industriel et un horizon possible de valorisation commerciale du travail entrepris (Cochoy, 20II), via la poursuite d'au moins quatre objectifs : enrichir l'information des consommateurs 
(connaissance des produits, informations inédites, garanties sur l'authenticité du produit), prendre en compte les évolutions stratégiques du marché mondial du vin (Barrère, 2007), anticiper l'évolution programmée de la réglementation européenne en matière d'étiquetage des vins, améliorer les pratiques vitivinicoles.

C'est la définition de la qualité du produit qui est ici en jeu. En effet, après avoir bâti, lors de la dernière réforme de l'OCM (Organisation commune de marché) une nouvelle segmentation de l'offre qui distingue les vins à indication géographique dont les AOP (ex-AOC) et les IGP (ex-vins de pays), des vins sans indication géographique, la Commission européenne demande, dans le cadre de la réforme des signes de qualité, de prouver pour les AOC désireuses d'obtenir l'AOP, leur lien au terroir, et pour les ex-vins de pays souhaitant décrocher un IGP, leur lien au territoire. Le passage des AOC aux AOP s'accompagne donc de la rédaction de nouveaux cahiers des charges qui devront être validés par la Commission européenne en 2014. La contrepartie en étant une protection de ces indications géographiques au plan international (Galbrun, 20ıo). L'enjeu de l'écriture du lien au terroir est une réflexion sur la nature de ce lien et la redéfinition des conditions de son expression dans les vins. La définition de la qualité constitue, ainsi, un point sensible de la coordination par le marché.

Le modèle conventionnaliste est, nous semble-t-il, approprié pour aborder l'enjeu de cette définition. En effet, l'organisation de la production, les modalités d'élevage du vin, les investissements effectués, les modalités de commercialisation du produit, l'étiquetage des bouteilles, etc., doivent être en cohérence avec une vision commune de ce qui fait la qualité du produit. Stanziani (2005) a mis en évidence l'hétérogénéité des conventions permettant de définir un produit alimentaire et les différentes échelles d'évaluation. En replaçant notre analyse dans le cadre général de l'apport des conventionnalistes, on abordera la qualité comme une construction sociale endogène contribuant à coordonner l'activité des acteurs, au même titre que les prix. Le système juridique, en institutionnalisant et en concrétisant cette convention de qualité - issue des pratiques de la société - en assure la diffusion et la stabilisation.

Avant de passer à l'examen de l'évolution juridique, posons quelques jalons théoriques permettant de penser les conventions de qualité. 


\subsection{Conventions et conventions de qualité}

\subsubsection{Convention : représentation collective extériorisée}

En suivant la tradition des économistes des conventions (Dupuy et al., I989; Eymard-Duvernay et al., 2006), nous pouvons aborder les conventions comme cadres d'interprétation dans lesquels les acteurs sont contraints d'inscrire leurs actions - registre d'actions possibles, harmonisation des comportements (Doeringer et Piore, I97I) et cadres des représentations et des anticipations (Keynes, $1936^{6}$ ) - et en même temps résultats de l'action des différents protagonistes du secteur du vin - construction par les acteurs qui les mobilisent et les interprètent (Weber, 1995). Revenons sur ces deux aspects.

La convention est un cadre pour la décision des agents, pour leurs actions et leurs interprétations. Chez Lewis, ce cadre est un savoir commun. Il est aussi réducteur de doute. Pour les conventionnalistes aussi, les conventions " limitent une forme d'incertitude non probabilisable menaçant les marchés financiers et régissent des anticipations " (Boyer et Sylvestre, I986, p. 9). Il en est de même pour le marché vitivinicole. L'incertitude sur la qualité devra être surmontée. Deux types d'incertitudes existent (Karpik, 1996, 2007) : incertitude sur la qualité du bien, incertitude sur la qualité du service échangé. La première peut renvoyer à l'incertitude sur le goût, on n'est pas sûr "d'avoir le bon goût » (Barrère, 2007); par exemple, les notes émises par Robert Parker sont censées dire ce qu'est le bon goût en matière de grands vins de Bordeaux (Chauvin, 20II). La seconde incertitude renvoie à l'opportunisme qui peut pousser les producteurs à modifier les caractéristiques du vin. Ainsi, les règles qui gouvernent l'étiquetage en France trouvent leur source dans le mouvement de lutte contre les fraudes qui agita le monde du vin au tournant du $\mathrm{XX}^{\mathrm{e}}$ siècle (Stanziani, 2005). La convention désigne donc " l'organisation sociale au travers de laquelle

6 Notamment le chapitre I2. C'est J.M. Keynes (1936) puis P.B. Doeringer et M.J. Piore (1985) qui vont donner pour la première fois un rôle analytique important à la coordination par les conventions. Keynes définit la convention comme une adjonction au fonctionnement des marchés grâce à leur pouvoir d'harmonisation des anticipations ; Doeringer et Piore comme un substitut au fonctionnement des marchés, grâce à leur pouvoir d'harmonisation des comportements. Pour une discussion sur ces conventions, on peut voir, notamment, Favereau (I989). 
la communauté se dote d'une référence commune, produit une représentation collective extériorisée qui fonde les anticipations individuelles " (Orléan, I989, p. 244). Dit autrement, la convention " renseigne les opérateurs sur ce qui constitue, pour une période donnée, les formes légitimes de production et d'échange. La convention met donc en œuvre une représentation normative du développement économique " (Salais et al., I986).

La convention est aussi le résultat d'actions individuelles et collectives. En ce sens, les comportements définissent une convention : elle est une régulation dans les comportements ou dans les comportements et les représentations (Lewis, 1969). Une telle définition de la convention permet de l'appréhender comme une " coordination par le précédent " (Favereau, 1982) : on se réfère au comportement antérieur tant qu'il n'est pas prouvé que cela soit erroné. L'état présent de l'opinion est supposé « correct » jusqu'à preuve du contraire (Keynes, 1936).

La transformation est donc liée au sentier d'évolution (Arthur, 1989; David, 20oI) ; pour comprendre l'évolution de la convention de qualité, il est nécessaire de connaître l'histoire du secteur du vin, "l'histoire compte " (David, 20oI). Cette dépendance au sentier peut impliquer une irréversibilité et l'émergence d'un paradigme technico-économique (Freeman et Perez, 1988). En effet, les règles européennes cherchent à améliorer l'information du consommateur, la compétitivité du marché du vin et la construction d'un marché unique du vin en Europe, en accentuant les exigences en matière de traçabilité et de définitions des liens aux origines (territoires et terroirs). En essayant de devancer la réglementation, les viticulteurs vont mettre en place des systèmes de géotraçabilitét. Ceux-ci vont entraîner une innovation-produit : les producteurs sont persuadés qu'audelà de l'information fournie aux consommateurs, une connaissance plus fine des vins manipulés (du sol, du climat, des cépages, etc.) permettra d'améliorer les savoir-faire (Cochoy, 20II). Cette innovation-produit aura un impact sur les innovations de procédés (qui impliquent une diversité au niveau des routines de production), les innovations organisationnelles (qui impliquent une diversité au niveau des routines de fonctionnement) et une évolution de la demande (le comportement et l'apprentissage des consommateurs pouvant être modifié) (Yildizoglu, 20II). En définitive, la

7 Dont Geowine est un exemple parmi d'autres. 
dynamique de l'économie prend alors la forme d'une série de coévolutions qui va modifier la pression sélective à chaque niveau : routines, operational rules, produits, firmes, secteurs, voire pays.

Il semblera difficile à l'avenir de revenir sur l'exigence d'information en matière de qualité et d'origine que demande la réglementation. De même, les pratiques viticoles induites par ces évolutions, la nature cumulative des connaissances sur lesquelles se basent les innovations structurent le développement technologique et induisent des " lock-in " (Arthur, 1989). On pourra déceler des saillances de marché (Schelling, 1960). La légitimité de celles-ci reposera alors sur la façon dont les acteurs vont se les approprier, vont leur donner sens au cours de leurs activités, vont les considérer comme une réalité provisoirement incontournable. Les systèmes de géotraçabilité peuvent être étudiés comme une volonté de mettre en place une institution saillante du marché du vin ${ }^{8}$.

La convention est donc cadre et résultat des actions. Elle a un caractère dual, en même temps restrictif, elle limite l'éventail des choix, et ouverture du champ des possibles (Orléan, 1994) ${ }^{9}$. Nous pouvons voir ici un écho aux notions d'encastrement et de découplage : " l'encastrement des activités économiques dans les relations sociales (encastrement au sens de Granovetter), la construction de leur autonomie relative (découplage au sens de White, performation au sens de Callon), mais aussi la façon dont les activités économiques peuvent engendrer des liens sociaux dont les relations interpersonnelles ne constituent qu'une forme parmi d'autres " (Cochoy et Grossetti, 2008, p. 6). Chaque acteur (dans notre exemple, chaque régulateur, consommateur et amateur de vin, producteur, coopérative, cave, etc.) participe à un milieu institutionnel où il est, au sens fort, acteur sur le mode de l'apprentissage et de l'exercice. "Une participation à une norme déjà déposée dans le monde, mais qui, bien que déjà là, ne se manifeste que dans l'investissement subjectif dont elle fait l'objet " (Karsenti, 2005, p. 183). Une norme déjà là mais qui ne prend son sens que dans son usage. L'acteur participe activement, c'est-à-dire non pas en s'affirmant comme un sujet, mais en agissant en sujet. "L'ordre des causalités entre le social et l'économique devient circulaire, ou plutôt la société et

8 Comme peuvent l'être aussi les notes établies par Robert Parker (cf. Chauvin, 20II).

9 Il n'y a pas que les conventionnalistes à avoir insisté sur ces deux aspects, on peut voir aussi, entre autres, North (1990) et Aoki (200I). 
l'économie cessent d'être des substances préalables pour devenir le résultat des échanges » (Cochoy et Grossetti, 2008 p. Io).

\subsubsection{Qualité du vin : métissage de conventions marchandes et domestiques}

La convention légitime qui sera retenue est le résultat d'un arbitrage entre plusieurs conventions de qualité qui peuvent coexister. Plus précisément, trois catégories de conventions de qualité permettent d'évaluer les biens sur les marchés (Boltanski et Thévenot, 1987, 1991 ; Thévenot, 1989 ; EymardDuvernay, 2002) : une convention industrielle qui renvoie à la conformité à un standard, à un investissement technique qui assure une régularité temporelle et une irréversibilité ; une convention domestique qui s'appuie sur des savoir-faire spécifiques, sur le rôle coordinateur de la confiance et de la tradition ; une convention marchande dont le prix est l'étalon de la qualité. Dans le cas de produit de qualité spécifique, Sylvander (1995) montre que ce sont principalement les conventions marchandes et domestiques qui sont mobilisées et qui fonctionnent sur deux segments distincts également importants : un modèle de nécessité (consommateurs populaires, réguliers, avertis) qui se fonde sur une convention marchande ; un modèle de loisir (consommateurs aisés, occasionnels et novices) qui se base sur une convention domestique.

Dans la convention de qualité marchande, la coordination est résolue par les prix. Ceux-ci expriment l'importance des désirs convergents. Parce que chaque agent est censé être capable de payer un prix pour une bouteille, il est possible d'estimer la valeur du vin. Le modèle d'évaluation est l'allocation optimale grâce aux mécanismes de marché ; celui-ci est le plus à même d'évaluer la qualité du vin. Cette convention sera donc concrétisée par des règles permettant le jeu concurrentiel : normes de protection de la santé, informations générales sur le produit (composition, calories, produits allergènes, etc.), développement des marques. Le vin est un bien économique comme les autres. La convention marchande doit assurer la qualité du service échangé en contrant les comportements opportunistes, notamment frauduleux.

Dans la convention de qualité domestique, la qualité est moins précise. Pour la définir, il faudra avoir recours à la tradition, au savoir-faire, à l'histoire, à l'origine. Le vin n'est pas un bien comme les autres, il peut être aussi considéré comme un patrimoine. La convention domestique doit 
assurer la qualité du bien en instaurant la confiance dans le temps. Cette convention sera donc concrétisée par des règles permettant la confiance et notamment l'instauration de marques collectives (AOP ou IGP par exemple), par le développement de l'information sur le processus de production, l'origine des produits, les règles d'élevage des vins.

\subsection{Le droit comme résultat des délibérations sur les conventions légitimes}

Comme nous venons de le voir, une convention " désigne le dispositif constituant un accord de volonté tout comme son produit doté d'une force normative obligatoire " (Dupuy et al., 1989, p. I43) ; en outre, plusieurs conventions de qualité peuvent coexister. Le système juridique illustre cette force normative et devra arbitrer entre des évaluations reposant sur des critères différents. Ainsi, l'activité économique est perçue comme ancrée dans le droit et non comme un espace autonome de calcul, perturbé par le droit. "Les autorités de régulations ne peuvent être réduites à des règles rationnelles, optimisées suivant des critères neutres, par exemple des mécanismes d'incitation. Elles sont des lieux de délibération sur les conventions légitimes. Il résulte de ce positionnement une articulation étroite avec le droit, comme espace majeur de délibération sur les conventions légitimes»(Eymard-Duvernay, 2006, p. I5) ${ }^{\mathrm{IO}}$.

L'approche interprétative des conventions permet de penser un droit réflexif. Le système juridique est, comme la convention qu'il outille, un modèle de comportement et ne se limite pas à une prescription obligatoire (Reynaud, 1992) ${ }^{\mathrm{II}}$. Son caractère incomplet ${ }^{\mathrm{I2}}$ va traduire la part des acteurs dans son interprétation et sa construction (Isla, 2006). C'est une représentation de l'ordre juridique proche de la sociologie du droit dans la tradition wébérienne : rapports actifs entretenus par les acteurs sociaux avec les dispositions juridiques.

Io L'économie des conventions a toujours porté un intérêt au droit (voir par exemple Thévenot, I992; Favereau, 1989).

II Nous sommes proches de l'institutionnalisme proposé par Commons (I934) et Veblen (I898) : les institutions sont un ensemble de working rules qui stabilisent la contradiction entre le conflit et la coopération.

I2 La " texture ouverte du droit " pour reprendre une expression des juristes (par exemple Jeammaud, I982). 
Les acteurs vont s'emparer de la réglementation et en tireront des effets différents puisqu'ils projettent sur l'organisation du marché du vin (OMV) des projets et des objectifs différents. Entre le cadre juridique et les acteurs du secteur, le rapport est un rapport de participation (Descombes, 2004 ; Karsenti, 2005). Par exemple, la mise en place de systèmes de géotraçabilité est un enjeu pour les producteurs. Le défi est pour eux de s'approprier l'objectif marchand-concurrentiel énoncé par les règles pour le digérer, le renouveler et le transformer en objectifs nouveaux : créer du lien avec le consommateur; donner du sens à l'AOP ; transformer le consommateur en amateur compétent et lui donner le sentiment qu'il entre dans le cercle des connaisseurs de vin ; fédérer le consommateur par les enjeux sur la qualité du vin (terroir...) et au-delà sur la protection de la nature et l'éthique du travail, sur la durabilité du marché du vin. L'enjeu est aussi de participer à la production de normes dans le cadre de l'autorégulation du marché demandée par le régulateur. En résumé, il s'agit, dans le cadre d'un secteur où l'augmentation quantitative des $\mathrm{AOP}$ depuis plusieurs dizaines d'années a conduit à une uniformisation des vins, uniformisation accentuée par l'entrée en crise de ce secteur depuis la fin des années 1990 (Barrère, 2007), de participer à la création de ces liens sociaux que sont les règles, pour les orienter, dans la mesure du possible, vers ses propres objectifs. Même les règles les plus " techniques ", i.e. les moins "interprétables " a priori, sont porteuses de valeurs, nourries d'une interprétation du social et de conceptions précises du mode de régulation envisagé (Lascoumes et Le Galès, 2005). Elles coordonnent les représentations sur les comportements (Aliprantis, I980; Jeammaud, I982; Morin, 199I) et participent à la construction d'une identité collective (Searle, I998).

Il y a accord sur un critère supérieur d'évaluation unanimement accepté à l'intérieur d'un espace géographique et temporel donné. Ces jugements locaux vont s'ancrer progressivement dans des dispositifs collectifs qui leur permettent de circuler dans des espaces de plus en plus larges (DubuissonQuellier et François, 20II) ; en étant mobilisés par des acteurs extérieurs au collectif de départ, ces jugements gagnent en généralité.

L'évolution du droit incorpore de fait une dépendance vis-à-vis du chemin. "Les évolutions et adaptations conceptuelles s'empilent les unes aux autres, et la structure de pensée juridique qui en résulte à un instant donné contient les formes qui, bien que supplantées en un certain sens, n'en continuent pas 
moins de dessiner le sentier d'évolution du droit » (Deakin, 2006, p. 238). La mise en perspective historique permet d'identifier, dans les évolutions passées, les éléments qui participent au sentier d'évolution.

En matière vitivinicole, la tradition européenne est de loin la plus riche et la plus étendue dans le monde. Dans cette tradition, le cadre réglementaire français a une place particulière car il est historiquement le premier à avoir été développé, il va de ce fait inspirer le cadre européen. Cette prégnance du cadre institutionnel français apparaît dès la fin du XIX ${ }^{e}$ siècle.

Au milieu du XIX ${ }^{\mathrm{e}}$ siècle, la viticulture joue un rôle majeur dans le développement économique de la France, mais le phylloxéra détruit les vignes dans les années 1870 et 1880 . Les producteurs commencent alors à utiliser des substituts artificiels. Face à ce comportement opportuniste, le gouvernement français produit la première législation du vin en i889. De nombreux autres décrets pour prévenir les fraudes vont suivre pendant vingt ans. Les producteurs français commencent à utiliser des plants de vigne hybrides plus résistants aux maladies, mais de telles vignes procurent des vins de faible qualité. Pour cette raison, les producteurs mélangent souvent leurs vins aux vins algériens qui ont un degré d'alcool plus élevé ; cela produit rapidement une surproduction et une baisse des prix. En voulant lutter contre cette situation, les producteurs réintroduisent des vins frauduleux et artificiels. Le gouvernement français intervient finalement avec des règlements forts en 1907 conçus pour diminuer la surproduction et pour lutter contre la fraude. En même temps commencent à se développer dans certaines régions, des associations de viticulteurs qui cherchent à garantir l'authenticité de leurs productions. Le législateur s'inspire de ces pratiques et leur donne un ancrage plus général en créant, dans la loi de 1905, " les appellations d'origine " pour plusieurs produits (vin, moutarde de Dijon, fromage de Roquefort, cidre, bière...). Les labels sur le vin définissent les aires de culture, les variétés de grappes, les pratiques œnologiques, le degré d'alcool, le rendement productif, l'irrigation, etc. En I927, la France interdit les vignes hybrides et régule les types de grappes requises pour le vin d'appellation. La loi du 30 juillet 1935 crée le système de " Protection et Désignation d'Origine ». Cette loi est la base de la régulation des régimes de qualité européens qui suivra. En 1943, le gouvernement français crée une nouvelle appellation sous la dénomination "vins spécifiques de qualité supérieure ». Cette loi combine plusieurs régulations précédentes : elle 
restreint la production non seulement aux origines spécifiques (à travers la délimitation de zones) mais aussi selon des critères de production spécifiques comme la variété des grappes, le minimum d'alcool et le maximum de rendement du vignoble. De plus, est établi le "Comité National des Appellations d'Origine ", une branche du gouvernement conçue pour administrer les processus des AOC pour des vins de haute qualité. Cette évolution de la réglementation française montre un empressement à imposer des informations spécifiques quant à chaque étape de la chaîne de production (aires cultivées, produits ajoutés, pratiques employées). Ceci est, même si les mots ne sont pas encore utilisés, une régulation de la traçabilité et de la géotraçabilité dans laquelle le terroir et l'origine sont des aspects majeurs.

Lors de la signature du Traité de Rome en 1957, les productions de vin française et italienne sont comparables et représentent plus de la moitié du vin mondial (Smith et al., 2007). L'Italie, à l'opposé de la France, a une réglementation très peu poussée (le gouvernement italien ne voit pas la nécessité de restreindre son industrie : il n'y a pas de régulation des prix ni de restriction sur les plantations, seulement des baisses de taxe pour la distillation du surplus de vin et des restrictions quant aux importations provenant de l'extérieur de la CEE). Dès les années I960, les institutions communautaires essaient de réguler ce secteur en créant une organisation commune du marché (OCM) spécifique au vin. L'OCM s'inspire des régulations qui existent dans les États membres et en particulier de celle de la France.

En 1962 est rédigée 1'OCM vitivinicole fondatrice. Cette première régulation instaure une distinction entre deux catégories de vin : les vins de table (VDT) et les vins de qualité produits dans des régions déterminées (VQPRD). Pour définir cette dernière catégorie, les règlements communautaires s'inspirent largement du système français régissant les AOC. La deuxième OCM vitivinicole en I970 prolonge la première. Elle ne modifie pas les principes généraux énoncés en 1962, mais édicte des règles techniques précises. Ainsi, dès I970, des règles détaillées vont être établies au niveau européen : i) classement des vignes en variétés " recommandées, autorisées ou autorisées temporairement "; ii) classement plus précis des vins (VQPRD, VDT, vins aptes à donner du vin de table, vins pétillants, vins mousseux, vins de liqueur, moûts, sous-produits) ; iii) pratiques 
œnologiques (titre alcoolique naturel maximal, chaptalisation, degré d'enrichissement maximal, prestations viniques). Jusqu'au début des années 20ıo, la réglementation européenne reprend ce que la législation française avait déjà rédigé (Tinlot, 2007), tout en restant en deçà des exigences françaises en matière de qualité.

Ce rapide historique montre que la création du cadre juridique du marché vitivinicole a été l'objet d'une entreprise délibérée d'acteurs, d'« entrepreneurs institutionnels " (DiMaggio, 1988). Ceux-ci, constitués notamment des producteurs et du législateur français, sont davantage des acteurs empiriques dans un processus complexe et collectif que des démiurges tout-puissants qui créeraient seuls ces dispositifs institutionnels (ibid.). Ces " entrepreneurs institutionnels " participent à l'émergence de la convention de qualité du vin qui peut être opérationnalisée en deux temps, tout d'abord à travers une dimension institutionnelle - la convention constitue un repère cognitif commun à la grande majorité des acteurs du marché -, ensuite à partir d'une dimension pragmatique - la convention fait l'objet d'appropriations et provoque des effets différenciés contribuant à la solidifier.

\section{L'IMPORTANCE DES SAVOIR-FAIRE ET DE L'ORIGINE DANS LA CONSTRUCTION INSTITUTIONNELLE}

Nous venons de tracer un historique de l'évolution du cadre institutionnel vitivinicole, nous allons maintenant illustrer certains points de cette évolution en outillant notre analyse d'un logiciel de traitement statistique de texte. Nous considérons l'évolution des textes juridiques comme des marqueurs de l'évolution de la convention de qualitér ${ }^{3}$.

Pour mener à bien cette analyse, nous avons construit treize corpus juridiques, rassemblant les textes légaux élaborés entre 1960 et 2009, en

I3 Cette conceptualisation est congruente avec le pragmatisme proposé par Peirce puis Dewey. "Considérer quels sont les effets pratiques que nous pensons pouvoir être produits par l'objet de notre conception. La conception de tous ces effets est la conception complète de l'objet " (Peirce, I879, § I5). Du point de vue pragmatique, un objet tire sa signification de ce qu'il deviendra, dans l'usage, donc de ses conséquents. 
tenant compte des comparaisons : France-Europe d'un côté et secteur vitivinicole-secteur agricole de l'autre ${ }^{\mathrm{I}}$. Pour la France, l'Europe et la France+Europe, nous avons construit trois corpus : traçabilité alimentaire et agroalimentaire (y compris le secteur du vin) ; organisation du marché du vin ; traçabilité alimentaire hors secteur du vin. Pour les trois textes fondamentaux du domaine (règlement CE I493/I999 portant sur l'organisation commune du marché vitivinicole, règlement CE 879/2008 portant sur l'organisation commune du marché vitivinicole et règlement CE 178/2002 établissant les principes généraux de la législation alimentaire et qui soumet une obligation de traçabilité à toutes les parties impliquées dans la chaîne alimentaire), nous avons construit un corpus avec tous les textes légaux mentionnés dans ces documents. Finalement nous avons créé un corpus avec les six Organisations Communes du Marché du vin (OCMV) et l'OCM agricole unique.

Le logiciel de traitement de texte que nous utilisons est le logiciel "Alceste ». Il permet de segmenter les corpus en mondes lexicaux retraçant les oppositions les plus importantes. Les diagrammes en arbre (les dendrogrammes) retracent, pour chacun des corpus, les mondes lexicaux décelables. Chaque monde lexical est représenté par les mots associés à une notion ou un concept, les plus caractéristiques par ordre décroissant de $\mathrm{Khi}^{2}$ associé. Chaque mot de ce type est celui que les rédacteurs des textes de la classe considérée ont associé à la gestion du secteur vitivinicole.

Les analyses du logiciel passent par plusieurs étapes :

- segmentation : segmentation de chaque corpus en groupes de mots ou Unités de Contexte Élémentaires (UCE). Chaque UCE contient le même nombre de $\operatorname{mots}^{15}$ en fonction de la taille du corpus. Par exemple, le corpus européen sur l'organisation du secteur du vin comporte 36 textes et 5 64I UCE. Celles-ci contiennent en moyenne I9,2 mots pleins ;

- lemmatisation : réduction des mots à leur racine ;

I4 Cf. liste des corpus en annexe.

I5 Sont exclus de ce recensement ce que les concepteurs du logiciel (cf. Reinert, 200I, Benzécri, 198I) appellent les "mots vides", i.e. les mots qui ne sont pas reliés à un concept ou une notion et qui ne servent qu'à relier les notions entre elles (conjonctions, articles, etc.). 
- tableau de contingence : les mots sont inscrits en colonne et les UCE en ligne ;

- double classification descendante hiérarchique pour obtenir des classes stables caractérisées par leurs principaux mots (test du Khi²);

- description des classes en appliquant l'analyse de correspondance et la classification hiérarchique.

À partir de cette analyse textuelle, nous pouvons faire émerger plusieurs caractéristiques que nous avons entrevues dans la première partie ${ }^{16}: d^{\prime}$ 'une part, l'impact de la réglementation française dans la réglementation européenne depuis les années I960; d'autre part, les contraintes qui s'orientent dans le sens d'une uniformisation de la PAC et d'une volonté de construire un " marché unique » du vin ; ce processus d'unification de l'industrie du vin démarre dès 1959 avec le Common Custom Tariff ; enfin, les potentialités d'action et de création de solutions que ce cadre porte. En effet, les systèmes de géotraçabilité sont des réponses créatives qui sont apparues pour gérer l'incertitude et répondre, notamment, à la concurrence mondiale et au débat marques-AOP.

\subsubsection{L'empreinte française dans les textes européens}

Nous pouvons faire une première comparaison des textes français et européens en comparant les "mondes lexicaux ", i.e. les classes de mots de ces deux corpus. Grâce à une double classification descendante, le logiciel caractérise chaque monde lexical par ses principaux termes. Nous obtenons des dendrogrammes (figures I et 2) qui nous permettent de dégager deux premières caractéristiques des liens entre les réglementations françaises et européennes. D'une part, dans le cas de l'Europe, le $\mathrm{I}^{\mathrm{er}}$ monde lexical qui se distingue fait référence aux aspects techniques liés au vin (alcoométrie, volumique, ferment, moût, acidité...) alors que, pour les textes français, le $\mathrm{I}^{\mathrm{er}}$ monde lexical s'articule autour des notions de bassin vitivinicole, de canton, de commune, etc., la classe " technique " ne venant qu'en deuxième position. D'autre part, en ce qui concerne l'organisation du secteur vitivinicole, la législation française est de manière générale plus pointue et inspire la réglementation européenne sur de nombreux aspects tels que les contraintes liées aux questions de qualité et d'authenticité des vins produits

I6 Nous enrichissons ici les premières conclusions de Isla et Taljic (20I0). 
avec une AOC. La réglementation française est plus axée sur les aspects terroir, alors que la réglementation européenne se limite davantage à la définition technique du vin. Dans les deux cas, bien sûr, l'origine est liée à l'appellation et aux critères géographiques, mais la législation européenne lie davantage l'origine à des questions de protection et de marque, alors que la législation française s'axe davantage sur des notions de producteurs et de qualité. Nous pouvons voir que ce n'est pas exactement la même conception de la qualité qui est mobilisée. Cette conception penche plutôt du côté de la convention marchande dans les textes européens, alors qu'elle est plus orientée vers une convention domestique dans les textes français. Cette distinction est toujours prégnante et revient sans arrêt en filigrane dans les débats nationaux, européens et mondiaux sur la qualité du vin (cf. infra).

Figure 1. Dendrogramme du corpus des textes français sur l'organisation et la traçabilité dans le secteur vitivinicole, 1960-2009

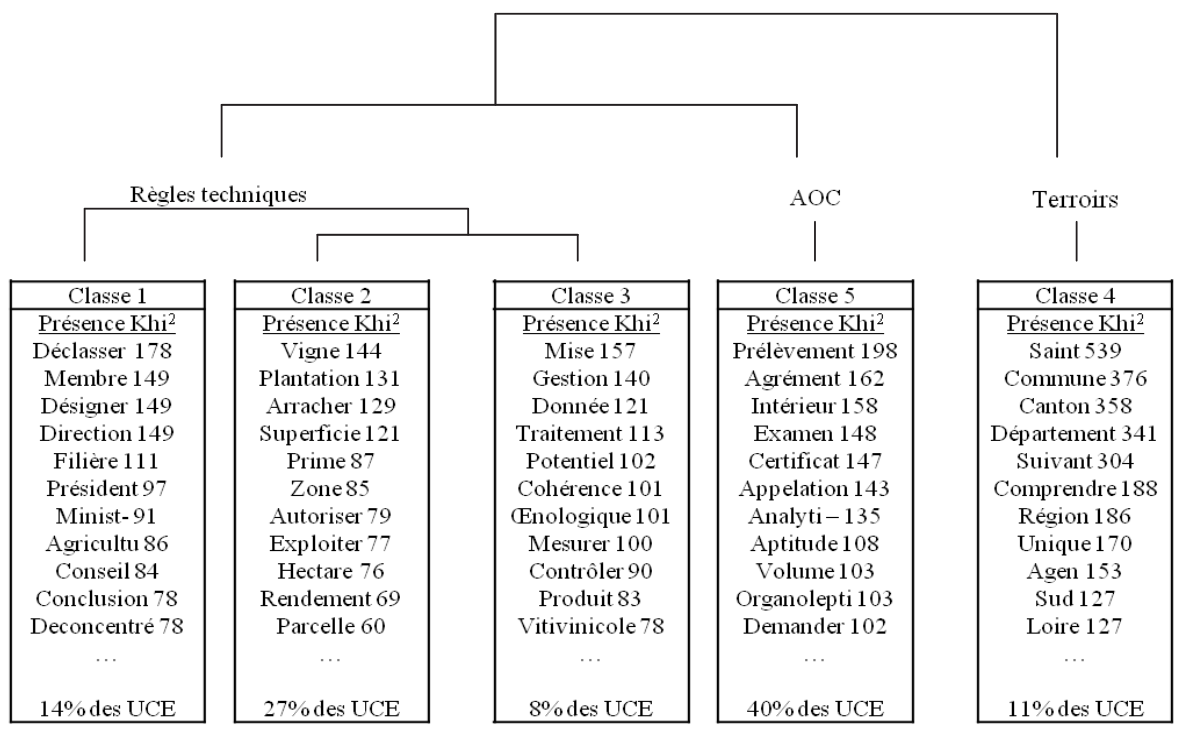

On a grâce aux dendrogrammes une vision d'ensemble des textes régissant le secteur. Il peut être aussi intéressant de regarder comment se forment, dans le temps, telle ou telle classe et faire une cartographie de cette construction. Si l'on procède à cette analyse la cartographie de la classe I du corpus européen est particulièrement intéressante parce qu'elle illustre ce que l'on avait pu deviner de la prise en compte de la qualité et du terroir ou de l'origine par les textes européens : une prise en compte récente et de plus en plus importante (cf. figure 3). 
Figure 2. Dendrogramme du corpus des textes européens sur l'organisation et la traçabilité dans le secteur vitivinicole, 1960-2009

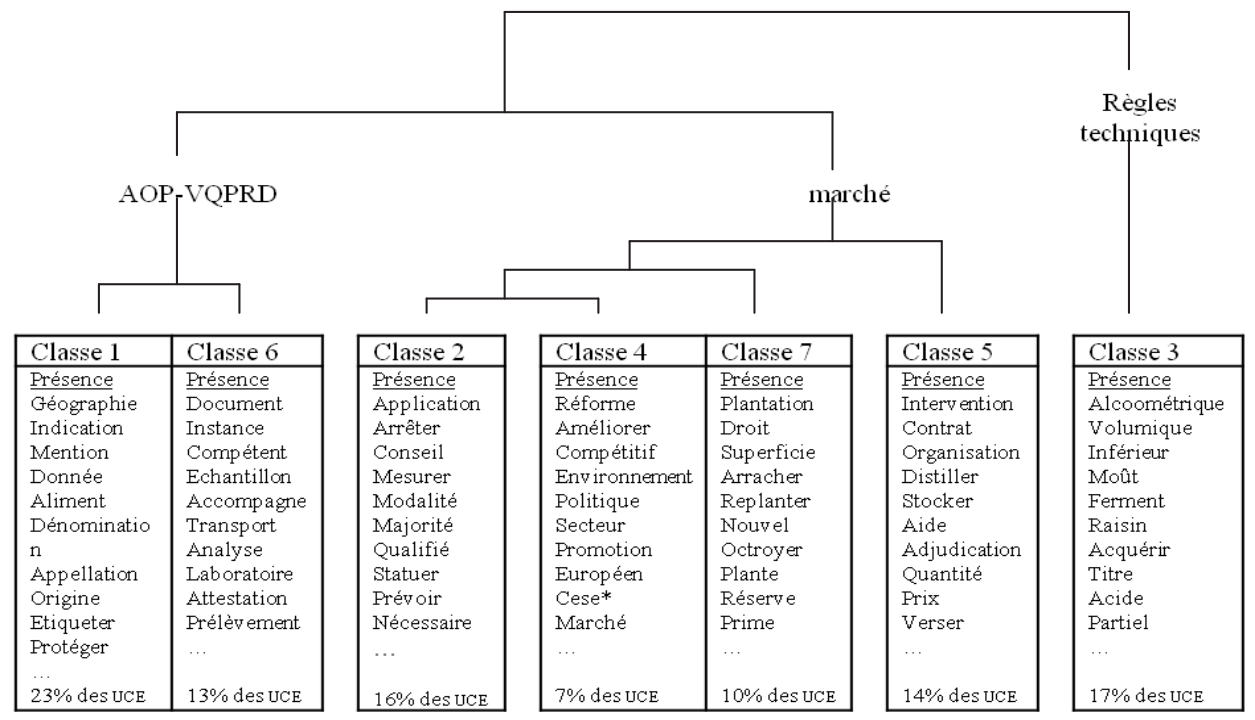

* Comité économique, social et environnemental

Dans la régulation des produits agricoles en général et du vin en particulier, il y a la volonté de contrôler toutes les étapes de la chaîne de production, c'est-à-dire une volonté de traçabilité. Nous l'avons déjà souligné, la nécessité de rendre transparent le processus productif est apparue dans le secteur du vin en raison des fraudes qui se sont développées à partir de la crise du phylloxéra. En outre, le développement de la traçabilité dans le secteur du vin n'est certainement pas complètement déconnecté de ce qui s'est passé dans le reste du secteur agroalimentaire, notamment avec les scandales liés à la santé des consommateurs (présence de benzène dans certaines bouteilles de Perrier en 1990, bouteilles de Coca-Cola contaminées au $\mathrm{COS}$ et au $\mathrm{H}_{2} \mathrm{~S}^{17}$ en Belgique dans les années 1990, crise de la vache folle dans les années I990, etc.). Ces crises ont poussé les acteurs publics et privés à s'orienter vers une économie de la qualité (Cazals, 2009). Pour tout le secteur agroalimentaire, et pour le secteur du vin en particulier, ce besoin de lutter contre la fraude et de protéger la santé du consommateur s'articule avec celui de développer la qualité pour ne pas perdre de part de marché (Barrère, 2007). On va donc avoir, au fur et à mesure que la réglementation se développe, un développement des aspects qualitatifs. Ce processus apparaît non seulement en raison de tous les objectifs cités, mais aussi par un

I7 Sulfure de carbone (COS) et Hydrogène sulfuré ( $\left.\mathrm{H}_{2} \mathrm{~S}\right)$. 
phénomène de dépendance de sentier, les aspects qualitatifs du vin ayant été mis en avant très tôt comme une solution pour gérer le secteur (1905, création des appellations d'origine pour le vin en France, cf. supra).

Nous allons utiliser la fonctionnalité du logiciel qui nous permet d'illustrer l'apparition chronologique et l'importance en évolution des concepts liés aux notions de désignation et d'origine du vin dans la réglementation européenne. Le logiciel peut faire une cartographie chronologique de chaque classe des dendrogrammes. Si nous faisons la cartographie de la classe I de la figure 2 ci-dessus, nous aurons l'illustration de l'apparition chronologique des concepts liés à la désignation d'origine et à la qualité des vins entre 1962 et 2009 (premier et dernier textes intégrés dans le corpus) en Europe. Sur l'axe des ordonnées, nous pouvons voir les numéros d'UCE (Unité de contexte élémentaire, i.e. fragment de texte). Nous pouvons faire apparaître, en " cliquant " sur n'importe quelle UCE, l'extrait de texte correspondant et le titre du texte juridique d'où provient l'extrait (nous en montrons un exemple dans la figure). Étant donné que les textes sont classés de façon chronologique, nous avons une trace " historique » de l'apparition d'une classe donnée ou d'un concept donné.

Figure 3. Carte chronologique des notions liées à la désignation et à l'origine dans la régulation européenne sur l'organisation et la traçabilité dans le secteur du vin (1960-2009)

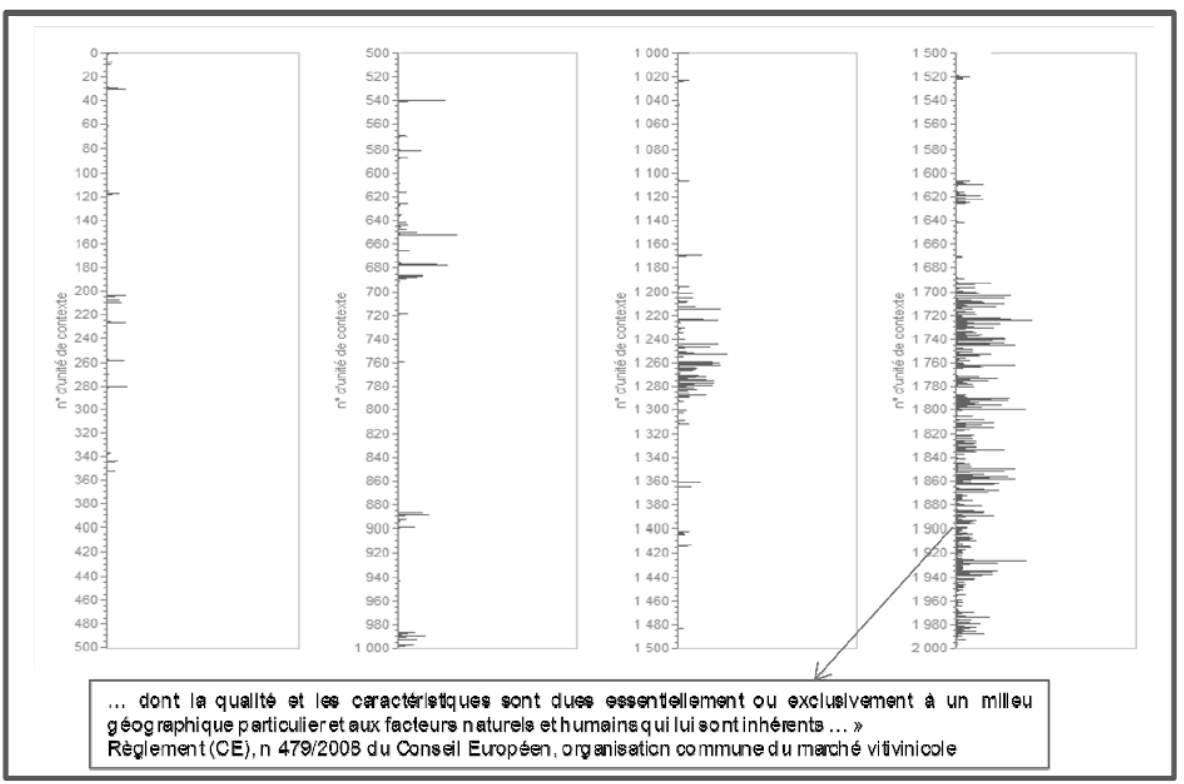


Nous avons ainsi voulu montrer que la qualité est de plus en plus prise en compte par les réglementations européennes. C'est un signe de la volonté de l'Europe de se placer dans le débat mondial AOP marques, nous y reviendrons plus loin ; c'est aussi un signe que l'Europe souhaite intervenir de plus en plus dans la définition des AOP (de la qualité, de la traçabilité), tandis que ce rôle a été principalement dévolu aux États jusqu'à présent. Cette évolution aura des conséquences sur le développement des systèmes de géotraçabilité qui, par définition, redéfinissent les liaisons entre la qualité, l'aire géographique de production et les pratiques productives et œnologiques. En effet, alors même que les exigences en matière de qualité s'accentuent, les acteurs de la filière vont percevoir et interpréter cette évolution en proposant des systèmes de géotraçabilité censés répondre et devancer l'évolution de la réglementation. Ce comportement n'est pas sans effet sur l'évolution de celle-ci, les régulateurs prenant en compte les pratiques des "régulés » dans la rédaction des nouvelles règles, comme les régulateurs français s'étaient inspirés en 1905 de ce qu'avaient mis en place certaines associations pour garantir l'authenticité de leur production viticole.

En définissant le terroir par la combinaison d'un nombre impressionnant de données (données climatiques et pédoclimatiques, stress hydrique, données géologiques, etc.), ces systèmes permettent de répondre aux exigences de la régulation tout en répondant à celles du producteur et du vigneron : améliorer la qualité de son vin, optimiser l'exploitation de sa parcelle, informer et séduire le consommateur (Isla, 2oII). Il s'agit d'une stratégie d'innovation des firmes vitivinicoles, déterminée par leur perception du caractère plus ou moins contraignant des réglementations existantes et par leurs anticipations quant à l'évolution future du cadre réglementaire (Oltra et Saint-Jean, 20II, p. 306).

En résumé, la $\mathrm{I}^{\mathrm{re}}$ OCM vitivinicole en 1962 instaure le principe général selon lequel il faut distinguer VQPRD et VDT, la gestion des premiers relevant de l'État et celle des seconds de l'UE. Pendant plusieurs décennies, les débats européens se focalisent sur la gestion de la quantité du vin produit et la suppression à terme de la production excédentaire. À partir des années I990, l'accent va aussi être mis sur les aspects qualitatifs du vin. L'émergence des notions liées au terroir et l'importance grandissante de ces notions illustrent cette volonté. Cette évolution va être marquée par 
le passage des VQPRD aux AOP lors de la rédaction de l'OCM unique (qui oriente les contraintes imposées aux acteurs dans le sens de la création d'un " marché unique " du vin, voire d'un " marché unique » agricole).

\subsubsection{Les enjeux d'une organisation commune du marché agricole : régulations du monde agricole et du secteur vitivinicole}

La volonté européenne de développer un marché concurrentiel et compétitif passe aussi par l'uniformisation ${ }^{18}$ de la réglementation agroalimentaire, c'est-à-dire par l'insertion progressive du secteur vitivinicole dans la politique agricole commune dans le cadre d'une réforme de la PAC. L'OCM vitivinicole de 2008 est la dernière propre à la filière ${ }^{19}$. On parlera désormais d'OCM unique pour tout le secteur agricole. Ceci traduit une évolution en trois points : i) les VQPRD et les VDT tombent désormais sous la qualification d'AOP ${ }^{20}$ et d'« indications géographiques protégées " (IGP) ; ii) l'étiquetage des vins et les pratiques œnologiques sont assouplis ; iii) les conditions favorables pour une autorégulation des opérateurs à l'égard de la gestion des AOP et des IGP sont développées.

Examinons les conséquences de cette évolution. Le premier point signifie qu'en matière de traçabilité, les exigences pour les autres produits alimentaires pourront être appliquées au vin. Dans le nouveau règlement adopté par le Parlement européen et le Conseil des ministres en automne 20II (règlement II69/20II, du Io octobre 20II), la Commission européenne

I8 L'« horizontalité communautaire » règlement (CE) n 479/2008, art. 923. Cette volonté s'inscrit dans un projet politique global relatif à la compétitivité de l'économie européenne : The Better Regulation Strategy. Lancée par le Conseil européen de Lisbonne en 2000 , cette stratégie comporte une liste d'objectifs transversaux tels que la «simplification » de la réglementation européenne (Mandelkern Group on Better Regulation, rapport final, I3 novembre 200I).

I9 Il y aura eu six OCM vitivinicoles : 1962, 1970, I979, 1987, I999 et 2008.

20 Il est intéressant de remarquer que, dans les années I960, l'expression « appellation d'origine " n'avait pas été retenue. Ceci correspondait à la situation des quatre pays viticoles de la Communauté européenne : la France et l'Italie avaient une législation des appellations d'origine, l'Allemagne et le Luxembourg avaient d'autres systèmes, la désignation "Vin de qualité produit dans une région déterminée » fut un compromis (Tinlot, 2007). 
propose l'étiquetage de calories et des ingrédients pour le vin afin d'informer davantage les consommateurs. L'analyse textuelle des textes jusqu'en 2009 nous permet d'entrevoir une telle évolution. Dans cette fusion des AOP agricoles, une autre tendance pourrait se dessiner. La construction institutionnelle du marché vitivinicole s'est en effet construite sur un consensus pour concevoir les VDT comme un problème public européen, tandis que les VQPRD relevaient de problèmes publics nationaux. Les États membres gardaient ainsi une marge de manœuvre ${ }^{2 \mathrm{I}}$ importante pour les VQPRD et pouvaient notamment établir des règles plus restrictives que celles exigées par la Commission. En se développant énormément depuis deux décennies, les VQPRD ont donc amenuisé le rôle des instances européennes dans la gestion du secteur ; mais en fusionnant les 2I OCM agricoles, la Commission ouvre la voie à un retournement de la situation, une prise de contrôle et un basculement des VQPRD, désormais AOP, vers un problème public européen. Il y a peut-être là une volonté des agents de la DG Agri de renforcer leur position dans la négociation vitivinicole en la raccordant à une réforme transversale du volet agricole, alors que jusque dans les années 2000 , les acteurs du secteur qui s'étaient investis dans les débats européens, l'avaient fait dans l'objectif de préserver la régulation de leur industrie d'une européanisation qu'ils pressentaient comme un risque (Smith et al., 2007).

Les deux autres points traduisent une volonté du régulateur de faire participer davantage les acteurs du secteur tout en assouplissant les pratiques œnologiques et l'étiquetage - l'utilisation des copeaux de bois, par exemple, est autorisée par 1'UE, alors que l'INAO en France l'interdit pour les AOP. Du fait de leur participation à la réforme, les professionnels sont mis dans une situation difficile et sont menacés par des évolutions qui pourraient être trop contraignantes. Ils doivent relever plusieurs défis, dont celui de la qualité. En travaillant sur le terroir dans une volonté d'optimiser l'utilisation des parcelles, les producteurs participant à des systèmes de géotraçabilité répondent à cet objectif. L'AOP n'est pas seulement un instrument pour améliorer la qualité, c'est aussi un outil marketing. L'assouplissement des pratiques œnologiques et surtout de l'étiquetage doit donc être intégré dans ce sens par les pratiques des professionnels. En matière d'étiquetage,

2I En France, l'évolution des règles concernant les appellations d'origine se fait essentiellement au sein de l'Institut national de l'origine et de la qualité (INAO), et plus précisément au sein de son Comité national des vins et eaux-de-vie. 
la règle était que, en dehors des mentions obligatoires (dénomination, titre alcoométrique, volume, $\mathrm{n}^{\circ}$ de lot de la bouteille, etc.), les mentions facultatives ne pouvaient figurer que dans la limite de ce qui était autorisé. Il existait une liste des mentions "facultatives réglementées " (nom de l'exploitation, cépage, millésime, mode d'élaboration, mentions traditionnelles). Tout ce qui n'était pas autorisé était interdit. Aujourd'hui, tout ce qui n'est pas interdit est autorisé, à condition que l'on puisse prouver la véracité des mentions. Cet assouplissement de l'étiquetage élargit grandement les possibilités mais aussi les défis pour les producteurs.

Le support informationnel proposé par le système de géotraçabilité donne au consommateur la possibilité de choisir l'information qu'il désire. C'est donc un outil marketing important puisqu'il est possible de mettre à la disposition du consommateur une quantité d'informations beaucoup plus importante quant à la qualité et à la provenance du vin en intégrant toute une série d'informations qui ne sont plus interdites et qu'il faudra pertinemment définir. Dans le cadre d'une autorégulation de plus en plus développée du secteur, l'enjeu de la construction d'un standard d'authentification devient donc décisif.

L'OCM unique ne bouleverse pas complètement le secteur a priori. En poursuivant ce qu'ont fait les règles antérieures, elle renforce le contrôle au sein des AOP. Sans introduire de nouveaux principes, elle ouvre la porte à des changements. Selon la façon dont elle sera mobilisée par les acteurs, elle pourra transformer le secteur en transférant le pouvoir au niveau européen au détriment des États, par exemple. D'autre part, les nouvelles règles incitent aussi les producteurs à s'en saisir pour innover en matière de relations avec les consommateurs, améliorer la qualité et l'information sur celle-ci, entre autres. L'OCM unique ouvre donc tout un champ des possibles. Son interprétation et sa mise en œuvre au sein des instances mondiales pourraient accentuer les changements, en profondeur, du secteur.

L'évolution des règles vers la prise en compte de plus de qualité liée à la localisation de la production et à l'amélioration des pratiques productives et œnologiques (i.e. la géotraçabilité) (comme nous l'avons montré avec les dendrogrammes et la cartographie) nous conduit vers un troisième aspect du cadre institutionnel : l'ouverture des possibles, et en l'occurrence la réponse créatrice aux pressions mondiales qui peuvent intervenir dans le secteur du vin. 


\subsubsection{Le marché vitivinicole européen dans le marché mondial : le débat AOP-marques}

Depuis 1995, l'Organisation Mondiale du Commerce (OMC) intervient dans la régulation du secteur. Celle-ci ne porte que sur un nombre limité d'enjeux - lutte contre l'usurpation de noms, définition du vin, délimitation des pratiques œnologiques autorisées - mais peut avoir des répercussions importantes dans l'organisation du secteur. Les institutions européennes concernées sont également soumises à des pressions importantes pour une réforme des OCM, au nom de la limitation du coût de l'exception agricole européenne dans les négociations de 1'OMC. Smith, de Maillard et Costa (2007, p. 9I) avancent " l'hypothèse selon laquelle le trait le plus marquant de l'évolution de l'univers vitivinicole européen est la fin de l'hégémonie des institutions européennes, et surtout françaises, au sein de la régulation mondiale du vin».

Nous avons vu que les systèmes de géotraçabilité développés par les acteurs représentent une réponse aux contraintes imposées par la régulation européenne et une façon de devancer ce que les acteurs perçoivent de cette évolution, tout en approfondissant le lien avec le consommateur. Le développement des informations sur la qualité et l'origine a aussi un autre aspect : c'est un outil (ouverture des possibles) que vont pouvoir utiliser les acteurs mais aussi les rédacteurs des règles européennes pour construire leur argumentation dans le débat mondial de l'organisation du secteur vitivinicole.

Au sein de l'OMC, la volonté d'établir un marché plus compétitif relance régulièrement le débat toujours latent entre les tenants des marques et ceux qui souhaitent un vin AOP. Il y a ceux qui, comme la France et plus globalement les pays européens, ont toujours défendu une qualité de leurs produits liée à un savoir-faire et à un terroir, à travers les indications géographiques et les appellations d'origine - il est intéressant de remarquer que, dans la législation française sur le vin, le mot " marque " n'est pas présent - ; et ceux qui, dans une volonté acharnée de maintenir une concurrence au sens le plus libéral du terme, défendent une approche en termes de marques commerciales arguant que les AOP ou les IGP induiraient des distorsions de concurrence et fausseraient l'information donnée au consommateur (Sylvander et Barham, 2009 ; Isla et Wallet, 2009). 
Pour l'instant, l'Europe défend sa vision au sein de l'OMC, mais les différentes initiatives des institutions européennes ne sont pas annonciatrices d'une régulation plus étroite ou plus forte. Au contraire, elles dénotent une "dé-spécification " de la politique vitivinicole en renvoyant à des règlements transversaux aussi bien en ce qui concerne l'étiquetage que le système des appellations d'origine ou la promotion du développement rural. Elles font référence aux normes internationales, dans une logique de subsidiarité qui jouerait en faveur de l'Organisation internationale de la vigne et du vin ${ }^{22}$ et des accords négociés dans le cadre de l'OMC. Les États membres restent responsables des aspects les plus conflictuels de la politique vitivinicole, notamment la gestion des situations de surproduction, alors que les outils qu'ils peuvent utiliser sont encadrés (Smith et al., 2007).

Ce débat AOP-marques s'inscrit aujourd'hui dans une situation de crise et une uniformisation de la façon de qualifier, voire d'étiqueter, le vin. D'un côté, les appellations se sont généralisées dans les différents pays, aussi bien dans " l'Ancien" Monde que dans le Nouveau. Spécificité de la viticulture française pendant de nombreuses années, les Espagnols avec les vins d'appellation d'origine et d'origine qualifiée - et les Italiens - avec les vins à dénomination d'origine contrôlée et contrôlée et garantie - ont adopté une classification similaire. En I999, apparaissent en Argentine les Indications de Provenance, les Indications géographiques et les "Denominación de origen controlada ", s'appuyant sur le modèle européen des AOP (Blanchy, 20I0). Les réglementations qui apparaissent en Argentine imposent en outre d'indiquer sur l'étiquette les cépages, le degré alcoolémique et la marque. En retour, ces pratiques sont diffusées en Europe et notamment en France, où certains producteurs créent des marques et indiquent des cépages. Tout ceci alors que les producteurs de VDT-IG français refusent la demande des producteurs AOP de faire figurer les cépages sur leurs étiquettes y voyant une atteinte à leur domaine réservé (Smith et al., 2007), tandis que la généralisation des AOP en France, depuis une vingtaine d'années, a banalisé cette marque collective. À ceci s'ajoute une remise en cause de la segmentation traditionnelle des marchés du vin par un modèle de marché de masse du goût qui se met en place, paradigme qui mine lentement mais sûrement

22 Dénommé « Office international de la vigne et du vin » de I924 à 200 . 
l'ancienne organisation des marchés (Barrère, 2007). Les marchés viticoles sont profondément affectés par ce mouvement de fond et les acteurs doivent donc trouver de nouvelles stratégies ${ }^{23}$. La mise en place d'un système de géotraçabilité est une illustration de cette volonté : se démarquer, procurer au consommateur l'information qui lui permette de distinguer les producteurs et les inscrire dans un paradigme aristocratique du goût, maintenir une définition de la qualité axée sur les savoir-faire et l'expérience.

En conclusion, nous pouvons voir qu'il s'agit pour les défenseurs des marques d'orienter la définition de la qualité vers une convention marchande. Les AOP et autres IGP, relevant de la convention domestique, distordent, selon eux, la concurrence. Au contraire, pour les acteurs européens, producteurs et régulateurs notamment, la définition s'inscrit dans une convention de qualité où le savoir-faire, la tradition, l'origine sont incontournables. Tout en cherchant à unifier le marché du vin, le régulateur européen s'engage à protéger les indications géographiques pour les producteurs qui prouveront leur lien au terroir (AOP) ou au territoire (IGP). Ainsi, le développement de la qualité liée à l'origine géographique et aux pratiques productives et l'information sur ces caractéristiques, i.e. la traçabilité géographique, est le point le plus marquant de l'évolution de la réglementation dans le secteur du vin. La régulation française y a joué un rôle majeur, de même que la perception que les acteurs ont pu avoir de son évolution qui les a conduits à développer des systèmes de traçabilité au-delà de ce que la réglementation demandait. Aujourd'hui, les contraintes européennes s'orientent dans le sens de la mise en place d'un " marché unique » du vin et en même temps donnent de nouvelles armes aux producteurs et aux négociateurs dans le débat mondial sur l'organisation du secteur du vin. L'enjeu est, pour les viticulteurs, la place du curseur entre convention marchande et domestique, dans la définition de la qualité vitivinicole.

23 Pour une approche détaillée des différentes configurations et des différents sentiers d'évolution possibles des marchés de vin de haute qualité (" marché aristocratique ") compte tenu des caractéristiques informationnelles desdits marchés, on peut se référer à Barrère (2007). 


\section{RÉFÉRENCES}

ALIPRENTIS N. (1980). La place de la convention collective dans la hiérarchie des normes, Paris, Librairie Générale de Droit et de Jurisprudence, préface de H. Sinay, 363 p.

AOKI M. (200I). Toward a Comparative Economic Analysis, Cambridge, Massachusetts Institute of Technology Press.

ARTHUR B.W. (I989). « Competing Technologies, Increasing Returns, and Lock-In by Historical Events ", Economic Journal, 97, pp. 642-65.

BARRÈRE C. (2007). "The genesis, evolution and crisis of an institution: the protected designation of origin in wine markets ", Journal of Institutionnal Economics, vol. 3, $\mathrm{n}^{\circ} 2$, pp. I65-I8I.

BAZZOLI L. (2000). "Institutional Economics and the Specificity of Social Evolution: About the Contribution of J.R. Commons ", in F. Louca, M. Perlman (dir.), Is Economics an Evolutionary Science?, Cheltenham, Edward Elgar.

BÉLIS-BERGOUIGNAN M.-C., LÉVY R., OLTRA V., SAINT-JEAN M. (2OI2). « L'articulation des objectifs technico-économiques et environnementaux au sein des projets d'éco-innovations : le cas de la filière bois aquitaine ", Revue d'Économie Industrielle, $\mathrm{n}^{\circ} \mathrm{I} 38,2^{\mathrm{e}}$ trimestre, pp. 9-38.

BENZÉCRI J.-P. (I98I). Pratique de l'analyse des données : linguistique et lexicologie, Paris, Dunod.

BOLTANSKI L., THÉVENOT L. (1987). Les économies de la grandeur, Cahiers du CEE, série Protée, Paris, CEE-Presses universitaires de France.

BOLTANSKI L., THÉVENOT L. (I99I). De la justification. Les économies de la grandeur, Paris, Gallimard, $483 \mathrm{p}$.

BOYER R., SILVESTRE J.-J. (I986). "Introduction ", dans R. Salais, L. Thévenot (dir.), Le travail, marché, règles et conventions, Paris, INSEE-Economica.

BLANCHY G. (20II). "La construction des vins argentins à travers l'affichage ", Sciences de la société, $\mathrm{n}^{\circ} 80, \mathrm{pp} .6 \mathrm{I}-75$.

CALLON M. (2007). "What does it mean to say that Economics is Performative?", in D. MacKenzie, F. Muniesa, L. Siu (dir.), Do Economists make Markets? On the Performativity of Economics, Princeton, Princeton University Press, pp. 3II-357.

CAZALS C. (2009). "Qualités et innovations environnementales dans la viticulture et l'arboriculture fruitière : l'apport des mondes de production ", Revue d'Économie Industrielle, $\mathrm{n}^{\circ} \mathrm{I} 26,2^{\mathrm{e}}$ trimestre, pp. 3i-52.

CHAUVIN P.-M. (20II). " Extension du domaine de la note. Robert Parker comme saillance du marché des grands vins de Bordeaux ", dans P. François (dir.), Vie et mort des institutions marchandes, Paris, Presses de Sciences Po, pp. 79-IO7.

COCHOY F. (2OII). Sociologie d'un "curiositif ». Smartphone, code-barres 2D et self-marketing, Lormont, Éditions Le Bord de l'eau, coll. "Mondes marchands ", I77 p.

COCHOY F., GROSSETI M. (2008). " Autour de Harrison White : liens, marchés et nouvelles sociologies économiques ", Sciences de la Société, n 73, pp. 3-15.

COMMONS J.R. (I934). Institutional Economics. Its place in political economy, New York, MacMillan Compagny, reprint 1990, Transaction Publishers, vol. I et 2.

DAVID P.A. (200I). " Path dependence, its critics and the quest for "historical economics' ", in P. Garrouste, S. Ioannides (dir.), Evolution and Path Dependence in Economic Ideas, Cheltenham, Edward Elgar. 
DEAKIN S. (2006). "Les conventions du marché du travail et l'évolution du droit ", in F. Eymard-Duvernay (dir.), L'économie des conventions, méthodes et résultats, tome I, Paris, La Découverte, coll. "Recherches ", pp. 23I-247.

DESCOMBES V. (2004). Le Complément du sujet, Paris, Gallimard.

DEWEY J. (1938/1986). Logic: The Theory of Inquiry, New York, Holt, Rinehart and Winston, I938; aussi dans The Later Works, vol. I2, 1986.

DIMAGGIO P.J. (I988). "Interest and Agency in Institutional Theory ", in L.G. Zucker (dir.), Institutional Patterns and Organizations, Cambridge, Ballinger, pp. 3-32.

DOERINGER P.B., PIORE M.J. (1971/1985). Internal labor markets and Manpower analysis, $2^{\mathrm{e}}$ éd., Armonk NY, Sharpe.

DUBUISSON-QUELLIER S., FRANÇOIS P. (2OII). "Institutions économiques et institutions marchandes. Nature, puissance et genèse ", in P. François (dir.), Vie et mort des institutions marchandes, Paris, Presses de Sciences Po., pp. II-38.

DUPUY J.-P., EYMARD-DUVERNAY F., FAVEREAU 0., ORLÉAN A., SALAIS R., THÉVENOT L. (I989). "Introduction ", Revue économique, vol. 40, $\mathrm{n}^{\circ}$ 2, "L'économie des conventions ", mars, pp. I4I-I45.

EYMARD-DUVERNAY F. (2006). "Introduction », dans F. Eymard-Duvernay (dir.), L'économie des conventions, méthodes et résultats, Paris, La Découverte, coll. "Recherches ", tome 2, pp. II-I6.

EYMARD-DUVERNAY F., FAVEREAU O., ORLÉAN A., SALAIS R., THÉVENOT L. (2006). "Des contrats incitatifs aux conventions légitimes. Une alternative aux théories économiques néolibérales ", in F. Eymard-Duvernay (dir.), L'économie des conventions, méthodes et résultats. Tome 2. Développements, Paris, La Découverte, coll. "Recherches ", pp. 17-42.

FAVEREAU 0. (1982). Le niveau de l'emploi dans une économie en croissance, Thèse d'État en Sciences Économiques, Université de Paris I, novembre.

FAVEREAU 0. (1989). "Organisation et marché ", Revue Française d'Économie, vol. 4, n I, hiver, pp. 65-96.

FREEMAN C., PEREZ C. (I988). "Structural crises of adjustment, business cycles, and investment behavior ", in G. Dosi et al., Technical Change and Economic Theory, London, Pinter Press, pp. 38-66.

GALBRUN C. (20I0). "Origine et segmentation, un pari difficile ", Réussir Vigne, n I62, I5 avril, p. I7.

GRANOVETTER M. (1985). "Economic Action and Social structure: The Problem of Embeddedness ", American Journal of Sociology, nº 91, pp. 480-510.

ISLA A. (2006). " Dynamiques des référentiels économiques de l'action publique : participation des acteurs dans la définition des choix collectifs (illustration dans les secteurs publics de l'électricité et de l'eau) ", Cahiers du Gres, nº I4, avril.

ISLA A. (20II). "Étiqueter la traçabilité dans le secteur vitivinicole : répondre aux exigences conjointes du régulateur, du producteur et de l'amateur de vin ", Sciences de la Société, $\mathrm{n}^{\circ}$ 80, pp. 77-93.

ISLA A., TALJIC D. (20I0). " Le système de traçabilité Geowine : répondre aux enjeux de l'évolution du cadre institutionnel et du cadre réglementaire lié à la traçabilité vitivinicole ", rapport dans le cadre du Projet Geowine : géotraçabilité et viticulture, pôle de compétitivité Agrimip Innovation. 
ISLA A., WALLET F. (2009). "Innovations institutionnelles dans les dispositifs d'Indications Géographiques et intégration des principes de développement durable. Une approche par le concept de bien public ", Revue d'Économie Méridionale, Montpellier, France, vol. 57, $\mathrm{n}^{\circ}$ 225-226, pp. 9-36.

JEAMMAUD A. (1982). " Les principes dans le droit français du travail », Droit social, septembre-octobre i982, pp. 618-629.

JULLIEN B., SMITH A. (20II). "Conceptualizing the role of politics in the economy: Industries and their institutionalizations ", Review of International Political Economy, I8(3), pp. 358-383.

KARPIK L. (1996). " Dispositif de confiance et engagements crédibles », Sociologie du travail, 38(4), pp. 527-551.

KARPIK L. (2007). L'économie des singularités, Paris, Gallimard, 373 p.

KARSENTI B. (2005). " Du sujet en philosophie sociale », Esprit, $n^{\circ}$ 7, juillet, pp. I77-186.

KEYNES J.M. (1936/1977). Théorie générale de l'emploi, de l'intérêt et de la monnaie, Paris, Payot, $387 \mathrm{p}$.

LASCOUMES P., LE GALES P. (2005). "L'action publique saisie par ses instruments ", in P. Lascoumes, P. Le Galès (dir.), Gouverner par les instruments, Paris, Presses de Sciences Po, pp. II-4I.

LEWIS D.K. (I969). Convention: a Philosophical Study, Cambridge, Harvard University Press, $213 \mathrm{p}$.

MOATI P. (20I0). "La poussée des marques de distributeurs sur le marché alimentaire : interprétations et perspectives", Revue d'Économie Industrielle, $\mathrm{n}^{\circ} \mathrm{II}, 3^{\mathrm{e}}$ trimestre, pp. I33-I54.

MORIN M.L. (199I). Le droit de la négociation collective principe général du droit, Thèse pour le Doctorat d'État en Droit, Université des Sciences Sociales de Toulouse, janvier, IO33 p.

NORTH D. (1990). Institutions, Institutional Change and Economic Performance, Cambridge, U.K. and N.Y., Cambridge University Press.

OLTRA V., SAINT-JEAN M. (2OII). «Innovations environnementales et dynamique industrielle ", in M.C. Belis-Bergouignan, B. Jullien, Y. Lung, M. Yildizoglu (dir.), Industries, Institutions, Innovations : éléments de dynamique industrielle, Pessac, Presses universitaires de Bordeaux, pp. 297-346.

ORLÉAN A. (I989). " Pour une approche cognitive des conventions économiques ", Revue économique, vol. 40, $\mathrm{n}^{\circ} 2$ " L'économie des conventions", mars, pp. 24I-272.

ORLÉAN A. (dir.) (1994). Analyse économique des conventions, Paris, Presses universitaires de France, $403 \mathrm{p}$.

PEIRCE C.-S. (I879). "Comment rendre nos idées claires ", La Revue philosophique de la France et de l'étranger, 4 année, vol. 7, janvier, pp. 39-57.

REINERT M. (200I). "Alceste, une méthode statistique et sémiotique d'analyse de discours : application aux "Rêveries du promeneur solitaire" ", Revue française de Psychiatrie et de Psychologie médicale, vol. 5, $\mathrm{n}^{\circ}$ 49, pp. 32-36.

REYNAUD B., (1992). Le salaire, la règle, le marché, Paris, Christian Bourgois, coll. "Cibles XXI ", 2I5 p.

SALAIS R., BAVEREZ N., REYNAUD B. (I986). L'invention du chômage, Paris, Presses universitaires de France, coll. "Économie et liberté ", 27I p. 
SCHELLING T. (1960/1986). La stratégie du conflit, Paris, Presses universitaires de France, 312 p. (éd. originale : Strategy of Conflict, Cambridge MA, Harvard University Press, 1960).

SEARLE J.R. (1998). La construction de la réalité sociale, Paris, Gallimard, coll. " NRF essais", $303 \mathrm{p}$.

SMITH A., DE MAILLARD J., COSTA 0. (2007). Vin et politique (Bordeaux, la France, la mondialisation). Paris, Presses de Sciences Po.

STANZIANI A. (2005). Histoire de la qualité alimentaire (XIXe-XXe siècles), Paris, Seuil, 444 p.

SYLVANDER B. (1995). "Conventions de qualité, marchés et institutions : le cas des produits de qualité spécifique ", in F. Nicolas, E. Valceschini (dir.), Agroalimentaire: une économie de la qualité, Paris, INRA-Economica, pp. I67-182.

SYLVANDER B., BARHAM E. (2009). Geographical Indications and globalisation in agro-food supply chains, New York, Cabi Publishers.

THÉVENOT L. (1989). "Équilibre et rationalité dans un univers complexe », Revue économique, vol. 40, n 2, pp. I47-I98.

THÉVENOT L. (1992). " Jugements ordinaires et jugements de droit ", Annales Économies, Sociétés, Civilisations, $47^{\mathrm{e}}$ année, $\mathrm{n}^{\circ}$ 6, novembre-décembre, pp. I279-I299.

TINLOT R. (2007). " L'apport de la loi de I905 dans le droit communautaire : incidence du droit vitivinicole français sur la réglementation européenne ", in La loi du $1^{\text {er }}$ août 1905 : cent ans de protection du consommateur, Paris, La Documentation française, pp. I37-I45.

TORRE A. (2002). " Les AOC sont-elles des clubs ? Réflexions sur les conditions de l'action collective localisées, entre coopération et règles formelles ", Revue d'Économie industrielle, $\mathrm{n}^{\circ}$ Ioo, $3^{\mathrm{e}}$ trimestre, pp. 39-62.

VEBLEN T. (1898). "Why is economics not an evolutionary science? ", Quartely Journal of Economics, pp. 373-397.

WEBER M. (I995). Économie et société, tome i, Paris, Plon, 4Io p.

YILDIZOGLU M. (20II). "Approche évolutionniste de la dynamique économique ", in M.-C. Bélis-Bergouignan, B. Jullien, Y. Lung, M. Yildizoglu (dir.), Industries, Innovations, Institutions : éléments de dynamique industrielle, Bordeaux, Presses universitaires de Bordeaux. 


\section{ANNEXE - Liste des corpus}

Chaque texte est classé chronologiquement et identifié par une série de caractéristiques. Celles-ci sont reprises et inscrites dans un titre pour chaque texte. Par exemple, le titre " 0402*do_Viticole*ty_Dtve*d_I986*P_U*a_EC » signifie que le texte fait partie du $4^{\mathrm{e}}$ corpus : la régulation du secteur du vin, le type de document : directive, date : 1986, zone : Union européenne, auteur : Conseil européen. Chaque corpus est un ensemble des textes légaux élaborés entre 1960 et 2009.

Textes français :

I. Traçabilité alimentaire et agroalimentaire (dont secteur viticole)

2. Traçabilité dans le secteur du vin et organisation du secteur vitivinicole

3. Traçabilité alimentaire hors secteur viticole

Textes européens :

4. Traçabilité alimentaire et agroalimentaire (dont secteur viticole)

5. Traçabilité dans le secteur du vin et organisation du secteur vitivinicole

6. Traçabilité alimentaire hors secteur viticole

7. Textes juridiques européens cités dans le règlement CE/I493/I999 portant sur l'organisation commune du marché vitivinicole

8. Textes juridiques européens cités dans le règlement CE/879/2008 portant sur l'organisation commune du marché vitivinicole

9. Textes juridiques européens cités dans le règlement $C E / 178 / 2002$ établissant les principes généraux de la législation alimentaire et qui soumet une obligation de traçabilité à toutes les parties impliquées dans la chaîne alimentaire

Io. Les six OCM vitivinicole et l'OCM unique

Textes français et européens :

II. Traçabilité alimentaire et agroalimentaire (dont secteur viticole)

I2. Traçabilité dans le secteur du vin et organisation du secteur vitivinicole

I3. Traçabilité alimentaire hors secteur viticole 